Chinese Journal of Astronomy and Astrophysics manuscript no.

( $\mathrm{LT}_{\mathrm{E}} \mathrm{X}$ : ring.tex; printed on July 6, 2021; 19:12)

\title{
Tail emission from a ring-like jet: its application to shallow decays of early afterglows and GRB 050709
}

\author{
Yuan-Chuan Zou and Zi-Gao Dai \\ Department of Astronomy, Nanjing University, Nanjing 210093, China.
}

Received 2006 January 30; accepted

\begin{abstract}
Similar to the pulsar, the magnetic axis and the spin axis of the gamma-ray burst source may not lie on the same line. This may cause a ring-like jet due to collimation of the precessing magnetic axis. We analyze the tail emission from such a jet, and find that it has a shallow decay phase with temporal index equal to $-1 / 2$ if the Lorentz factor of the ejecta is not very high. This phase is consistent with the shallow decay phase of some early X-ray afterglow detected by swift. The ring-like jet has a tail cusp with sharp rising and very sharp decay. This effect can provide an explanation for the re-brightening and sharp decay of the X-ray afterglow of GRB 050709.
\end{abstract}

Key words: gamma rays: bursts $-\mathrm{X}$-rays: general

\section{INTRODUCTION}

It is well known that pulsars originate from the core collapse of massive stars. The average angle between the spinning axis and the magnetic axis of pulsars is about $27^{\circ}$ (Leahv, 1991). Similarly, the spin axis and magnetic axis of the central engine of the gammaray burst may not lie on one line. As the ejecta may be collimated by the magnetic axis, while the magnetic axis is processing, so the ejecta may be in a spiral shape at first(Fargion \& Grossi, 2005, and reference therein). As the diversity of the velocities of the ejecta [as assumed in the standard fireball model of gamma-ray bursts(Piran, 2005)], the spiral ejecta ejected at different times will collide and merge into one whole shell at last. These collisions just produce internal shocks of gamma-ray burst. At last, these collisions make the ejecta merge into a ring-shaped jet. Even if the the ejecta is conical, the baryon-loaded region still be ring-like (Eichler \& Levinson, 2003). Granot (2005) and

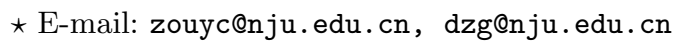


Eichler \& Granot (2005) have analyzed afterglows from ring-like jets. It has also been used to interpret the $h \nu_{\text {peak }}-E_{\text {iso }}$ relation(Eichler \& Levinson, 2004).

Tail emission plays an important role at the times when shocks disappear. The temporal index is $-(2+\beta)$ for a cone-shaped jet, where $\beta$ is the spectral index of the emission(Kumar \& Panaitescu, 2000; Yamazaki et al., 2005). Considering the zero point effect of time, the light curves can be steeper during a short period(Nousek et al., 2005: Zhang et al., 2005: Wu et al., 2006).

Nousek et al. (2005) and Zhang et al. (2005) have also shown that a shallow decay with index of about $-1 / 2$ follows the steep decay for most X-ray afterglows. For the Xray afterglow of short burst GRB 050709, there is an unexpected high-flux point followed by a very steep decay (Fox et al., 2005). These two observations can both be explained naturally by considering the tail emission of ring-like jets. In $\$ 2$ we give the expressions of tail emission from a ring-like jet. In $\S ₫ 3$ and 4 , the shallow decay and X-ray afterglow of GRB 050709 are analyzed respectively. At last, we summarize our results in 95

\section{MODEL}

Considering several ring-like sub-jets emitted from the central engine, they merge into one whole ring-like jet accompanied with internal shocks. This final ring with uniform energy density and sharp edges expands with Lorentz factor $\gamma$, as sketched in Fig. 11 Assuming the radiation from the ring-like jet begins and ceases at radius $R_{c}$ (and correspondingly at time $t_{c}$ ) suddenly, we calculate the tail emission from high latitudes of the ring. The relation is $R_{c} \simeq 2 \eta^{2} c t$, where $\eta$ is the mean Lorentz factor of the internal shocks.

The relation between the latitude angle $\theta$ and the observed time $t$ is

$$
R_{c}(1-\cos \theta)=c\left(t-t_{c}\right) /(1+z)
$$

where $z$ is the cosmological redshift. Neglecting the depth of the ejecta and the emission from time equal arrival surface, and defining the emissivity $I_{\nu^{\prime}}^{\prime}$ per unit area in the comoving frame, which is uniform in the whole ring, the flux density in the observer's frame is

$$
f_{\nu}\left(t>t_{c}\right)=\frac{I_{\nu^{\prime}}^{\prime}}{4 \pi D_{L}^{2}} \mathscr{D}^{2} \frac{\mathrm{d} S}{\mathrm{~d} t /(1+z)},
$$

where $D_{L}$ is the luminosity distance, $\mathscr{D}=1 /\left[\gamma\left(1-\sqrt{1-1 / \gamma^{2}} \cos \theta\right)\right]$ is the Doppler factor, and $\mathrm{d} S$ is the emitted area during a period $\mathrm{d} t$.

At early times when $\theta<\theta_{w}$, the tail emission is the same as the case of an on-axis conical jet, which has been investigated by many authors (Kumar \& Panaitescu, 2000; Fan \& Wei, 2005). There are two limiting cases: for $\theta \ll 1 / \gamma$,

$$
f_{\nu}\left(t>t_{c}\right) \propto \delta t^{0}
$$

and for $1 \gg \theta \gg 1 / \gamma$,

$$
f_{\nu}\left(t>t_{c}\right) \propto \delta t^{-(2+\beta)}
$$




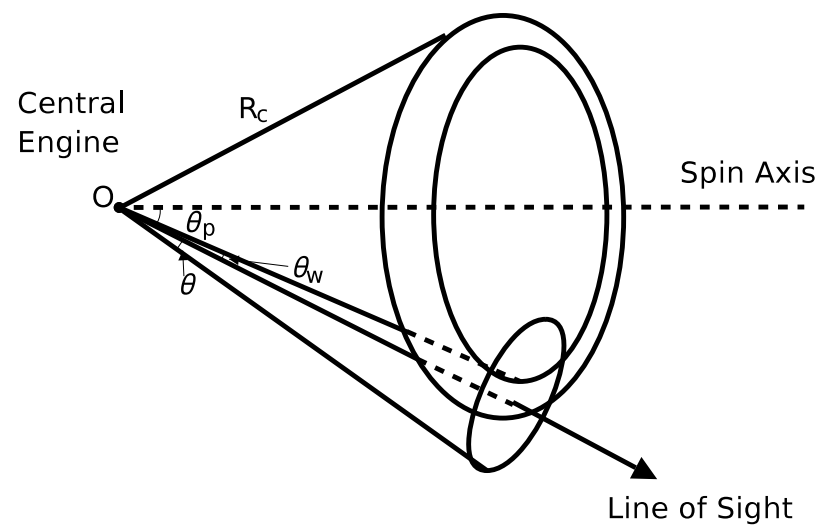

Fig. 1 Sketch of a ring-like jet at a distance $R_{c}$ from the central engine, where the main emission is just ceased, while an observer begins to receive the tail emission from high latitudes. $\theta_{p}$ is the half opening angle of the ring. $\theta_{w}$ is the half width angle. $\theta$ is the latitude of tail emitted region corresponding to the observed time $t$.

where $\delta t \equiv t-t_{c}$. Here we consider the emission as a single power-law profile $I_{\nu^{\prime}}^{\prime} \propto \nu^{\prime-\beta}$, which is valid for the high frequency emission $\nu^{\prime}>\max \left(\nu_{c}^{\prime}, \nu_{m}^{\prime}\right)$, where $\nu_{c}^{\prime}$ is the cooling frequency and $\nu_{m}^{\prime}$ is the typical frequency of synchrotron emission.

In the case $\theta>\theta_{w}$, the width of the ring can be neglected, and the flux density

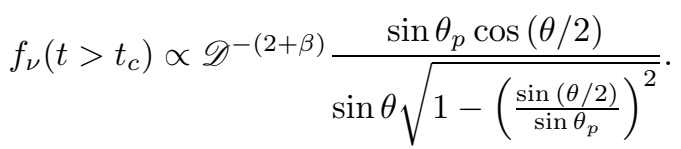

There are two limiting cases in which equation (5) can be simplified. For $\theta \ll 1 / \gamma$,

$$
f_{\nu}\left(t>t_{c}\right) \propto \delta t^{-1 / 2}
$$

and for $1 \gg \theta \gg 1 / \gamma$

$$
f_{\nu}\left(t>t_{c}\right) \propto \delta t^{-(5 / 2+\beta)}
$$

\section{SHALLOW DECAY OF EARLY X-RAY AFTERGLOW}

Statistics of the early X-ray afterglows has shown that there is a shallow decay phase with temporal index about $-1 / 2$ (Nousek et al., 2005; Zhang et al., 2005). This corresponds to the case: $1 / \gamma>\theta>\theta_{w}$, and can be described by equation (6), where the temporal index is just $-1 / 2$. As the general shallow decay lasts from $10^{2}-10^{3}$ s to $10^{3}-10^{4}$ s (Fig. 1 in Zhang et al., 2005), this gives limits: the Lorentz factor of the emitting shell $\gamma<7.3(1+$ $z)^{1 / 2} R_{c, 16}^{1 / 2} \delta t_{3.5}^{-1 / 2}$, and the width of the ring-like jet $\theta_{w}<1.4 \times 10^{-2}(1+z)^{-1 / 2} R_{c, 16}^{-1 / 2} \delta t_{2.5}^{1 / 2}$. 
(The conventional donation $Q=Q_{k} \times 10^{k}$ is used throughout this paper.) This implies that the shallow decay component originates from the shocked shells with low Lorentz factors, while these shocks may be formed due to ejected sub-shells with different Lorentz factors.

This model can answer the following questions:

Firstly, why is there a steep decay before the shallow decay appears in general case? In Zhang et al. (2005), it is general that the temporal index of this steep decay is less that -3 . The answer is that the two power law decays originate from two different emitting shells with different Lorentz factors. The steep decay corresponds to the greater Lorentz factor shell, which satisfies $1 / \gamma<\theta$, and the temporal indices are $-(2+\beta)$ or $-(5 / 2+\beta)$ corresponding to equations (44) and (7) respectively. The steep decay may become steeper because of the zero time selection effect( $\mathrm{Wu}$ et al., 2006).

Secondly, why is there no spectral evolution before and after the break time from the shallow decay phase to the steep decay phase. This is also mentioned with spectral index value $\sim-1$ in Zhang et al. (2005). It is believed that, after the break, the afterglow becomes a "normal" afterglow. It is possible that the tail emission phase and the "normal" afterglow emission phase are both in the case $\nu_{X}>\left\{\nu_{m}, \nu_{c}\right\}$ (corresponding to the spectral index $-p / 2$ ) and thus the spectra are the same.

Thirdly, since the shallow phase and the steep phase originate from different sources, how to understand the conjunction at the break time (also can be seen in Fig. 1 in Zhang et al., 2005)? As time goes on, the case converts from $1 / \gamma>\theta>\theta_{w}$ to $\theta>$ $1 / \gamma>\theta_{w}$, and then the light curve of the tail emission decay has a temporal index $-(5 / 2+\beta)$. This is steeper than the "normal" afterglow with temporal index $\sim-1.2$. Some time later, the "normal" afterglow will exceed the tail emission definitely, as in the case GRB 050525a (Fig. 1 in Nousek et al., 2005) (at about 3000s, there is a steep decay). However, GRB 050315 (Vaughan et al., 2005) can be classified into the case that "normal" afterglow exceed the shallow tail emission before the tail emission breaks to steep phase.

\section{X-RAY AFTERGLOW OF GRB 050709}

GRB 050709 is a short burst with duration $0.3 \mathrm{~s}$, and five points of X-ray emission after the burst were obtained by Swift and Chandra (Fox et al., 2005). Figure 2 shows the fit by assuming that the latter four points are the tail emissions from the first point, with parameters $R_{c}=7.7 \times 10^{16} \mathrm{~cm}, \gamma=15.5, \beta=1.1, \theta_{p}=0.5$ and $\theta_{w}=0.005$. As the first $\mathrm{X}$-ray point occurs at time about $100 \mathrm{~s}$, the radius $r \simeq 2 \eta^{2} \mathrm{ct} /(1+z) \simeq 5.2 \times 10^{16} \eta_{2}^{2} t_{2} \mathrm{~cm}$, is consistent with the value of the parameter $R_{c}$. As a short burst has less total energy than a long burst does, the ejected shell can be decelerated quickly. The Lorentz factor at $R_{c}$ is $\gamma \simeq E_{\mathrm{iso}} /\left(\pi R_{c}^{3} n m_{p} c^{2}\right) \simeq 26 E_{\mathrm{iso}, 50}^{1 / 2} n_{1}^{-1 / 2} R_{c, 16.5}^{-3 / 2}$, where the external medium density 


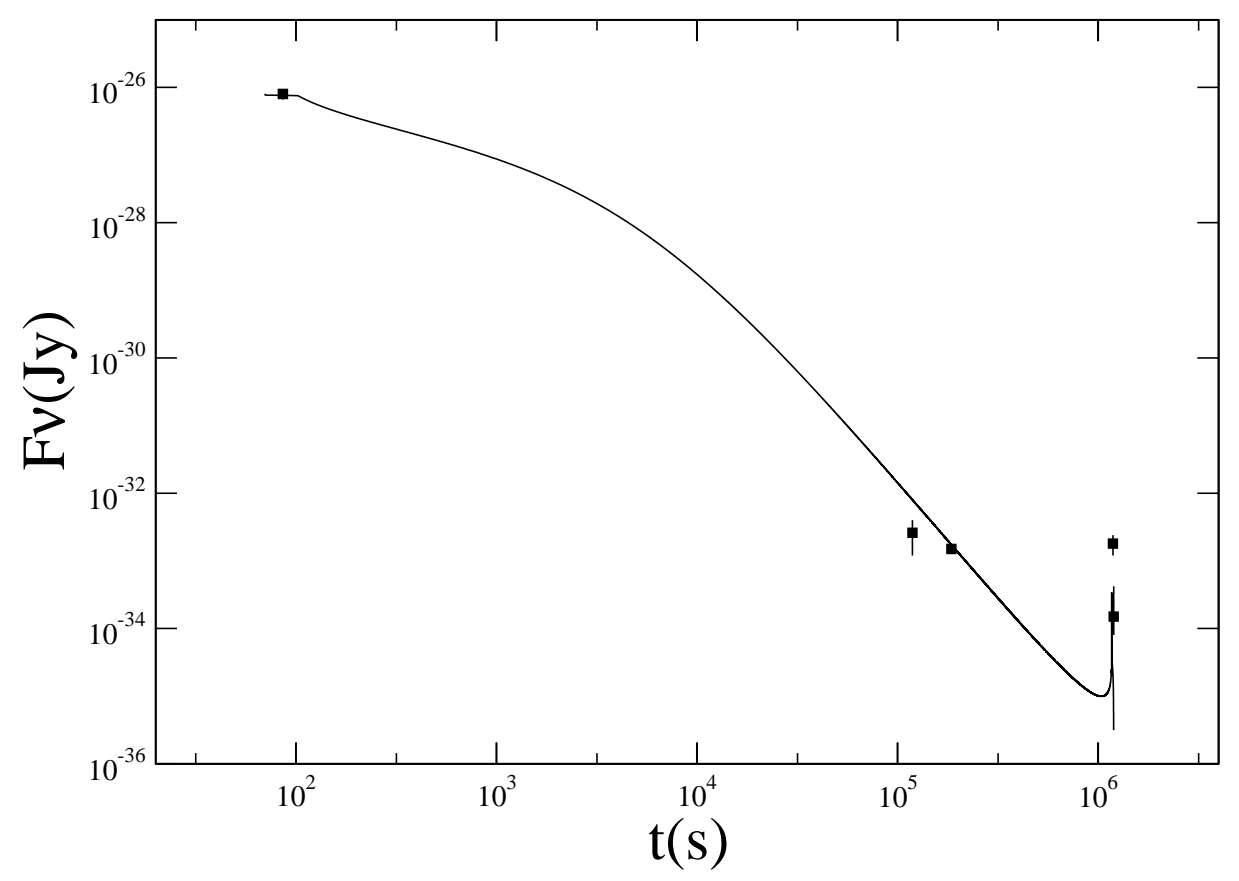

Fig. 2 Tail emission fit to the X-ray emission from GRB 050709. The five observed data are taken from Fox et al. (2005).

$n$ is chosen equal to $1 \mathrm{~cm}^{-3}$ because the host is a star-forming galaxy. Therefore, the parameters chosen to fit the X-ray data are reasonable for this short burst.

We can see four stages for this tail light curve: first, a horizontal phase corresponds to the case $\theta<\theta_{w}<1 / \gamma$; second, a shallow decay with temporal index $-1 / 2$ corresponds to the case $\theta_{w}<\theta<1 / \gamma$; third, a sharper decay with temporal index $-(2.5+\beta)$ corresponds to the case $\theta_{w}<1 / \gamma<\theta$; and finally, a tail cusp with sharp rising and very sharp decay, which comes from the end of the ring.

We should note that the solid line doesn't fit the data very well, especially that the tail cusp of the model can't reach to the observed data. This shortage may be overcome by considering a non-uniform ring-like jet or some other mechanism. However, its unique feature, which the emission after the tail cusp decays very sharply, is consistent with the last two observed points. On the other hand, it is possible that the second, third and fifth points in this figure belong to the "normal" afterglow from an external shock.

\section{CONCLUSIONS}

Enlightened from pulsars, we suggest that the magnetic axis and the spin axis of a gamma-ray burst source point to different orientations. The ejecta along the magnetic 
axis will form a ring finally. Gamma-ray emission will be observed if the observer locates in the solid angle of the ring. We have investigated the tail emission from a ring-like jet. We find that the early shallow decay phase and the late re-brightening of the X-ray emission of GRB 050709 can be explained.

Note that the shallow decay phase is only possible in the low Lorentz factor cases. For the case $1 / \gamma<\theta_{w}$, only the steep one appears. As the tail emission from the shells with high Lorentz factors decays very quickly, the main emissions will be dominated by the slower shells at later times.

YCZ thanks helpful discussions with Jia Wang and Xuefeng Wu. This work was supported by the National Natural Science Foundation of China (grants 10233010 and 10221001).

\section{References}

Eichler D., Granot J., 2006, ApJ, 641, L5

Eichler D., Levinson A., 2003, ApJ, 596, L147

Eichler D., Levinson A., 2004, ApJ, 614, L13

Fan Y. Z., Wei D. M., 2005, MNRAS, 364, L42

Fargion D., Grossi M., 2005, MNRAS submitted (astro-ph/0504638)

Fox D. B., Frail D. A., Price P. A., et al., 2005, Nature, 437, 845

Granot J., 2005, ApJ, 631, 1022

Kumar P., Panaitescu A, 2000, ApJ, 541, L51

Nousek J. A., Kouveliotou C., Grupe D., et al., 2005, ApJ in press (astro-ph/0508332)

Leahy D. A., 1991, MNRAS, 251, 203

Piran T., 2005, Rev. Mod. Phys., 76, 1143

Vaughan S., Goad M. R., Beardmore A. P., et al., 2006, ApJ, 638, 920

Wu X. F., Dai Z. G., Wang X. Y., et al., 2006, ApJ submitted(astro-ph/0512555)

Yamazaki R., Toma K., Ioka K., Nakamura T., 2005, MNRAS in press (astro-ph/0509159)

Zhang B., Fan Y. Z., Dyks J., et al., 2005, ApJ in press (astro-ph/0508321) 